\title{
Twig Pattern Matching: A Revisit
}

\author{
Jiang $\mathrm{Li}^{1}$, Junhu Wang ${ }^{1}$, and Maolin Huang ${ }^{2}$ \\ 1 School of Information and Communication Technology \\ Griffith University, Gold Coast, Australia \\ Jiang.Li@griffithuni.edu.au, J.Wang@griffith.edu.au \\ 2 Faculty of Engineering and Information Technology \\ The University of Technology, Sydney, Australia \\ maolin@it.uts.edu.au
}

\begin{abstract}
Twig pattern matching plays a crucial role in XML query processing. In order to reduce the processing time, some existing holistic onephase twig pattern matching algorithms (e.g., HolisticTwigStack [3], TwigFast [5], etc) use the core function getNext of TwigStack [2] to effectively and efficiently filter out the useless elements. However, using getNext as a filter may incur other redundant computation. We propose two approaches, namely re-test checking and forward-to-end, which can avoid the redundant computation and can be easily applied to both holistic one-phase and two-phase algorithms. The experiments show that our approach can significantly improve the efficiency by avoiding the redundant computation.
\end{abstract}

\section{Introduction}

The importance of fast processing of XML data is well known. Twig pattern matching, which is to find all matchings of a query tree pattern in an XML data tree, lies in the center of all XML processing languages. Therefore, finding efficient algorithms for twig pattern matching is an important research problem.

Over the last few years, many algorithms have been proposed to perform twig pattern matching. Bruno et al [2] proposed a two-phase holistic twig join algorithm called TwigStack, which breaks the query tree into root-to-leaf paths, finds individual root-to-path solutions, and merges these partial solutions to get the final result. One vivid feature of TwigStack is the efficient filtering of useless partial solutions through the use of function get Next. Later on several one-phase holistic algorithms (e.g., 3, ,5]) also use get Next to filter out useless elements. Using get Next as a filter can efficiently discard useless elements. However, get Next may incur other redundant computation. Li et al [4] try to resolve the redundant computation and propose TJEssential, but their approach involves much overhead and can not avoid some important types of redundant computation.

In this paper, we propose a different approach to avoid redundant computation, and this approach imposes less overheads and can be easily applied to both holistic one-phase and two-phase twig pattern matching algorithms that 


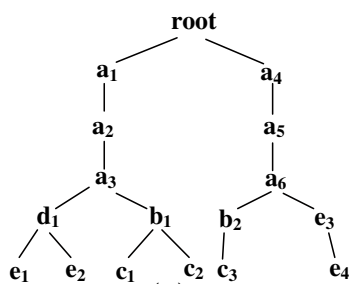

(a) $t$

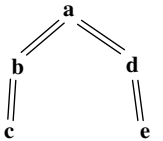

(b) $Q$

Fig. 1. Example data tree $t$ and tree pattern $Q$

are based on TwigStack. We present the algorithms TwigFast* and TwigStack* which extend TwigFast and TwigStack respectively by applying our proposed approach.

The rest of the paper is organized as follows. Section 2 provides background knowledge and recalls the major features of get Next and TwigFast. Redundant computation in getNext is explained in Section 3. Our approach for resolving redundant computation is presented in Section 4. The experiment results are reported in Section 5. Finally, Section 6] concludes this paper.

\section{Terminology and Notation}

An XML document is modeled as a node-labeled tree, referred to as the data tree. A twig pattern is also a node-labeled tree, but it has two types of edges: /-edge and //-edges, which represent parent-child and ancestor-descendent relationships respectively. The twig matching problem is to find all occurrences of the twig pattern in the data tree. For data trees, we adopt the region coding scheme [2]. Each node $v$ is coded with a tuple of three values: (v.start, v.end: v.level).

Below, we will use elements to refer to nodes in a data tree, and nodes to refer to nodes in a twig pattern. For each node $n$, there is a stream, $T_{n}$, consisting of all elements with the same label as $n$ arranged in ascending order of their start values. For each stream $T_{n}$, there exists a pointer $P T_{n}$ pointing to the current element in $T_{n}$. The function $\operatorname{Advance}\left(T_{n}\right)$ moves the pointer $P T_{n}$ to the next element in $T_{n}$. The function getElement $\left(T_{n}\right)$ retrieves the current element of $T_{n}$. The function $i s E n d\left(T_{n}\right)$ judges whether $P T_{n}$ points to the position after the last element in $T_{n}$. In addition, for node $n$, the functions $i s R o o t(n)$ (resp. isLeaf $(n)$ ) checks whether node $n$ is the root (resp. leaf), and parent $(n)$ (resp. children $(n)$ ) returns the parent (resp. set of children) of $n$. ancestors $(n)$ (resp. descendants $(n))$ returns the set of ancestors (resp. set of descendants) of $n$.

\section{Deficiencies in Previous Algorithms}

Many previous twig pattern matching algorithms use getNext of TwigStack to filter out useless elements. However, get Next may bring other redundant computation. In this section, we explain where the redundant computation comes 
Table 1. Example of redundant calls of getNext

\begin{tabular}{|c|c|c|c|c|c|}
\hline Step & getNext(a) & getNext(b) & getNext(c) & getNext(d) & getNext(e) \\
\hline \hline 1 & $\mathrm{a}\left(a_{1}\right)$ & $\mathrm{b}$ & $\mathrm{c}$ & $\mathrm{d}$ & $\mathrm{e}$ \\
\hline 2 & $\mathrm{a}\left(a_{2}\right)$ & $\mathrm{b}$ & $\mathrm{c}$ & $\mathrm{d}$ & $\mathrm{e}$ \\
\hline 3 & $\mathrm{a}\left(a_{3}\right)$ & $\mathrm{b}$ & $\mathrm{c}$ & $\mathrm{d}$ & $\mathrm{e}$ \\
\hline 4 & $\mathrm{~d}\left(d_{1}\right)$ & $\mathrm{b}$ & $\mathrm{c}$ & $\mathrm{d}$ & $\mathrm{e}$ \\
\hline 5 & $\mathrm{e}\left(e_{1}\right)$ & $\mathrm{b}$ & $\mathrm{c}$ & $\mathrm{e}$ & $\mathrm{e}$ \\
\hline 6 & $\mathrm{e}\left(e_{2}\right)$ & $\mathrm{b}$ & $\mathrm{c}$ & $\mathrm{e}$ & $\mathrm{e}$ \\
\hline 7 & $\mathrm{e}\left(e_{3}\right)$ & $\mathrm{b}$ & $\mathrm{c}$ & $\mathrm{e}$ & $\mathrm{e}$ \\
\hline 8 & $\mathrm{e}\left(e_{4}\right)$ & $\mathrm{b}$ & $\mathrm{c}$ & $\mathrm{e}$ & $\mathrm{e}$ \\
\hline 9 & $\mathrm{~b}\left(b_{1}\right)$ & $\mathrm{b}$ & $\mathrm{c}$ & $\mathrm{d}$ & $\mathrm{e}$ \\
\hline 10 & $\mathrm{c}\left(c_{1}\right)$ & $\mathrm{c}$ & $\mathrm{c}$ & & \\
\hline 11 & $\mathrm{c}\left(c_{2}\right)$ & $\mathrm{c}$ & $\mathrm{c}$ & & \\
\hline 12 & $\mathrm{~b}\left(b_{2}\right)$ & $\mathrm{b}$ & $\mathrm{c}$ & $\mathrm{d}$ & $\mathrm{e}$ \\
\hline 13 & $\mathrm{c}\left(c_{3}\right)$ & $\mathrm{c}$ & $\mathrm{c}$ & & \\
\hline
\end{tabular}

from. For ease of understanding, we use the query and data tree in Fig. 1 to exemplify the redundant computation of get Next. We present each step 1 of calling getNext over the root of the query tree (i.e., a) in Table 1

Basically, the redundant computation mainly comes from the following redundant test and late end.

Redundant test. redundant test is making redundant calls of get Next over some nodes in the query tree. The current elements of these nodes did not change in the previous step. Consider the data tree $t$ and query $Q$ in Fig. 1 . $a_{1}-a_{3}$ are self-nested nodes. After we found $a_{1}$ has a solution extension in step 1 , it is unnecessary to call get Next over the query trees rooted at the nodes $b$ and $d$ when testing whether $a_{2}$ has a solution extension. This is mainly because the current elements of the nodes $b, c, d$ and $e$ do not change during and after step 1 . This also happens on $a_{3}$ when checking if $a_{3}$ has a solution extension. Therefore, the calls of getNext over the nodes $b, c, d$ and $e$ in step 2-3 are redundant, and they are grayed in Table 1. For the similar reason, it is unnecessary to call get Next over the nodes $b, c, d$ and $e$ in step 4. This also happens in step 9 and 12 , and the calls of get Next over $d$ and $e$ are redundant. Furthermore, during step 5-7, the calls of get Next over the subtree rooted at node $b$ are redundant.

Late end. late end is wasting time on the elements that will not contribute to any solutions when some cursors of the streams reach ends. Suppose there are no elements to be processed in the stream of node $q$, it is possible to skip all the rest of the elements in the streams of nodes ancestors $(q)$ and descendants $(q)$. This can avoid some calls of getNext and the time spent on scanning the elements in some streams. For the example above, when there are no elements left in the stream $T_{d}$ after step 4 , we can directly set $P T_{a}$ to the end because the remaining elements in stream $T_{a}$ will not contribute to any solutions. Then, when we found the rest of the elements in the streams of descendant $(q)$ will not contribute any solutions, we can set the $P T$ pointers of these streams to the ends. In Table 1 . calls of get Next in step 8 and 13 are redundant and can be pruned.

${ }^{1}$ In this paper, a step is a call of get Next over the root of a query tree including the recursive calls. 


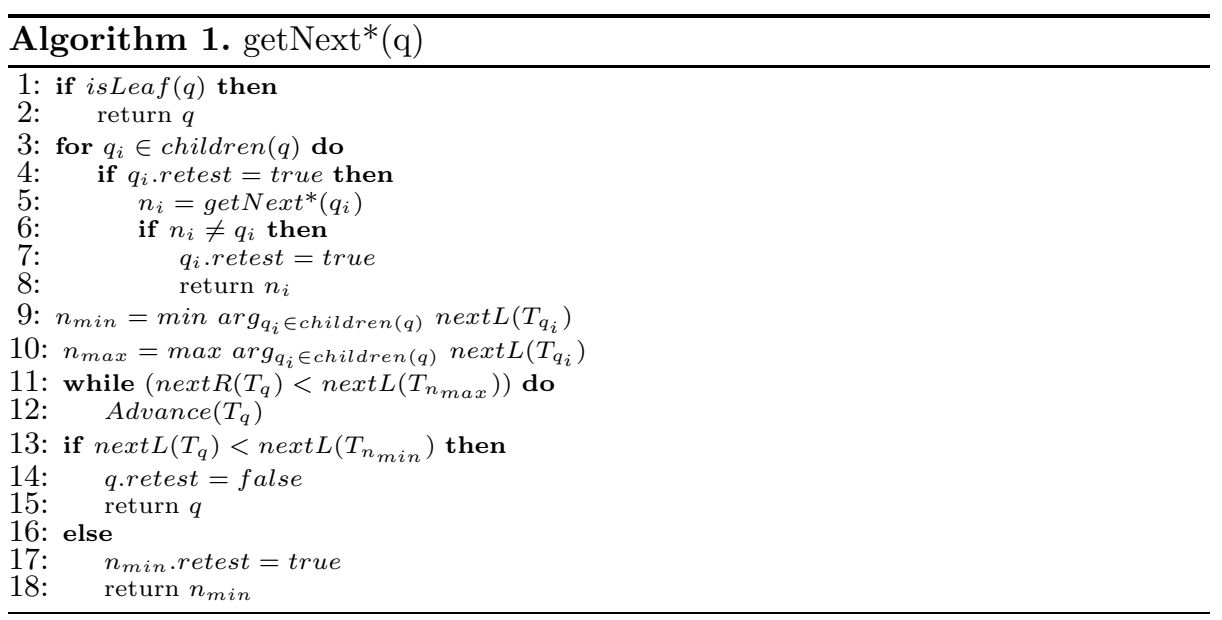

\section{Approach for Avoiding Redundant Computation}

\subsection{Re-test Checking}

Our solution for redundant test is called re-test checking and is mainly based on the following observation:

Observation. $\operatorname{get} N \operatorname{ext}(n)$ is used for testing whether a solution extension can be found for the current element of node $n$. Suppose get $N$ ext has been called over the node $n$ before. If the current element of any node in the query tree rooted at $n$ changes, it is necessary to call $\operatorname{get} N \operatorname{ext}(n)$ again for re-testing. Otherwise, $\operatorname{get} N \operatorname{ext}(n)$ does not need to be called.

We introduce an extra value retest for each query node to record whether getNext need to be called on this node in the next step. The initial value of retest is true, and this value is dynamic during computation. The new version of getNext is presented in Algorithm 1 .

get $N e x t^{*}$ has the following properties: (1) Given a query rooted at $Q$, get $N e x t^{*}$ is only called over the nodes whose value of retest is true, including the nodes that have not been tested by get $N e x t^{*}$ before and the nodes have been tested by get $N e x t^{*}$ but need to be tested again. (2) Suppose get $N e x t^{*}$ has been called over each node at least once. If $\operatorname{get} \operatorname{ext}^{*}(Q)$ returns a node $n$ in a step, get $N e x t^{*}$ will only be called over the nodes on the path from $Q$ to $n$ in the next step. The number of times getNext* is called will be bounded by the maximal depth of the query tree in the following steps.

With the properties above, the number of times get Next* is called can be significantly reduced, particularly when the query tree has many branches.

\subsection{Forward-to-End}

When the pointer $P T_{n}$ of the stream $T_{n}$ reaches the end, the rest of the elements in the streams of $n$ 's ancestors and descendants may become useless. Therefore, 

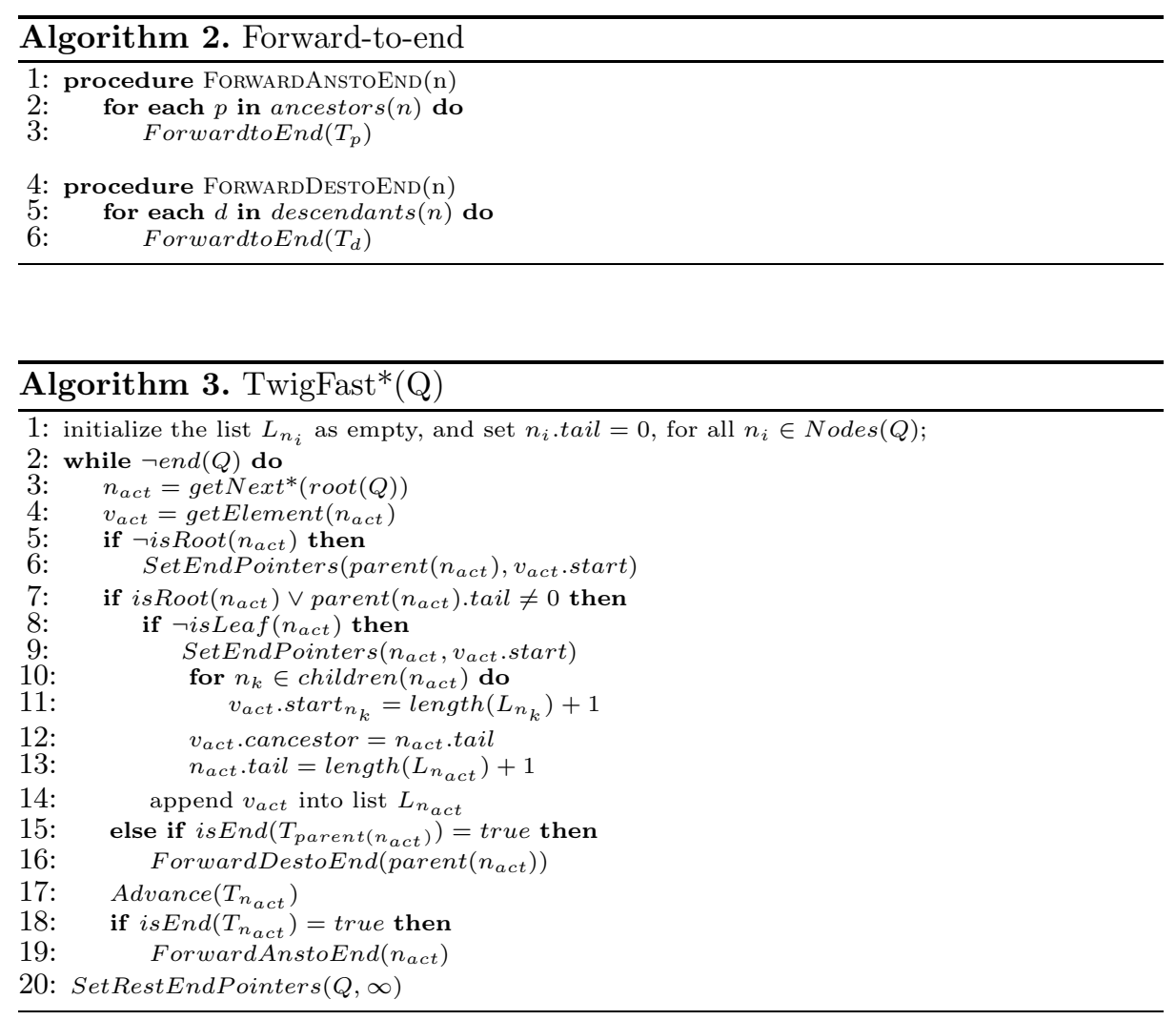

we need to find a solution to skip these useless elements. In our approach forward-to-end, we select two time points for skipping. Consider a query tree rooted at $Q$. Suppose $\operatorname{get} N \operatorname{ext}(Q)$ returns node $n$ in a step, and $P T_{n}$ reaches the end after calling Advance $\left(T_{n}\right)$. The two time points for skipping the rest of the elements in the streams of $n$ 's ancestors and descendants are as follows:

Time point 1 . We immediately skip the rest of the elements in the streams of $n$ 's ancestors after calling $\operatorname{Advance}\left(T_{n}\right)$ because we can not find any solution extension for them in the following steps.

Time point 2. We can not immediately skip the rest of the elements in the streams of $n$ 's descendants after calling Advance $\left(T_{n}\right)$ because they are still potential elements that may contribute to final solutions. We have to wait until there are no elements in the stack $S_{n}$ for the two-phase algorithms that use stacks for storing intermediate results and all the end positions in the list $L_{n}$ have been set for the one-phase algorithms that use lists for storing final results.

The pseudocode of skipping the rest of the elements in the streams of the node $n$ and $n$ 's ancestors and descendants is shown in Algorithm 2 . 


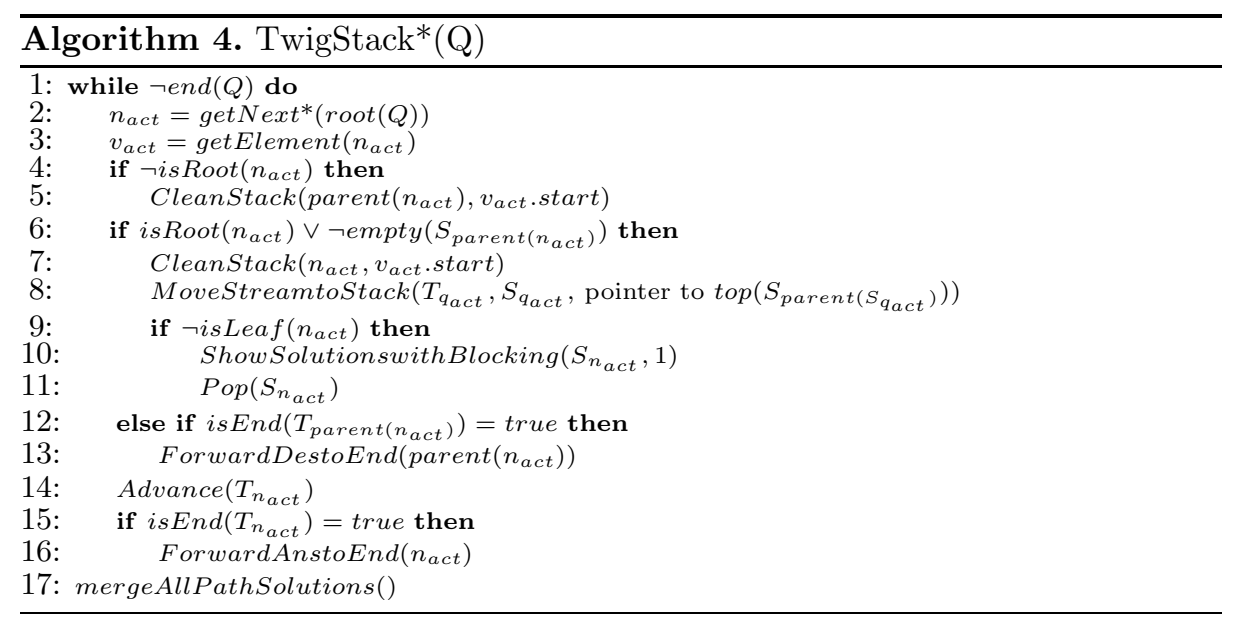

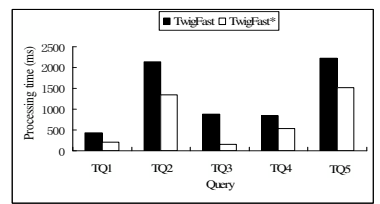

(a) low frequency on nonleaf nodes

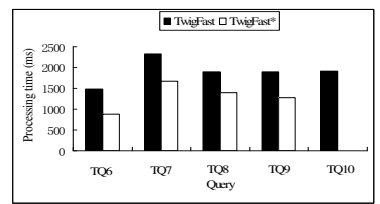

(b) low frequency on leaf nodes

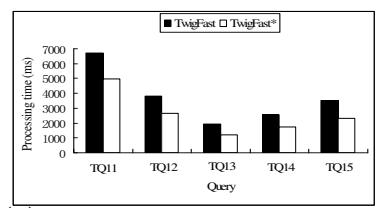

(c) all nodes with high frequencies

Fig. 2. Processing time of queries with different characteristics

\subsection{TwigFast* and TwigStack*}

Algorithm 3 and 4 extend the algorithm TwigFast and TwigStack respectively by applying re-test checking and forward-to-end.

\section{Experiments}

In this section, we present the experiment results on the performance of TwigFast* against TwigFast [5] and TwigStack* against TwigStack [2] and TJEssential [4, with both real-world and synthetic data sets. The algorithms are evaluated with the metrics of processing time. We selected the queries with different characteristics for more accurate evaluation.

We implemented TwigFast*, TwigFast, TwigStack*, TwigStack and TJEssential in $\mathrm{C}++$. All the experiments were performed on $1.7 \mathrm{GHz}$ Intel Pentium M processor with $1 \mathrm{G}$ RAM. The operating system is Windows 7 . We used the following three data sets for evaluation: TreeBank [1, DBLP [1] and XMark [6]. The queries for evaluation are listed in Table 2, which contain '//' and '/' edges.

Performance of answering the queries with different characteristics.In order to make the experiments more objective, we selected the queries with differ- 
Table 2. Queries over TreeBank, DBLP and XMark

\begin{tabular}{|c|c|c|}
\hline Data set & Query & XPath expression \\
\hline TreeBank & TQ1 & ///V//S \\
\hline TreeBank & TQ2 & //ADV $[/ \mathrm{S}][/ \mathrm{PP}] / / \mathrm{NP}$ \\
\hline TreeBank & TQ3 & //A//S//VP \\
\hline TreeBank & TQ4 & //ADJ[//NN]//DT \\
\hline TreeBank & TQ5 & //VP//ADV[//VP][//NP]//S \\
\hline TreeBank & $\overline{\mathrm{TQ} 6}$ & ]//S[/NP]//CONJ \\
\hline TreeBank & TQ7 & //NP[//NP][//PP]//_NL \\
\hline TreeBank & TQ8 & //S//VP/NP]//_HASH_ \\
\hline TreeBank & TQ9 & //S[/ADV][//PP]/NP \\
\hline TreeBank & TQ10 & ///VP[//NP][//PP]//FILE \\
\hline TreeBank & TTQ11 & ]//VP $[/ \mathrm{NP} / / \mathrm{NP}][/ \mathrm{S}[/ / \mathrm{PP}] / \mathrm{VP}] / / \mathrm{NN}$ \\
\hline TreeBank & TQ12 & //NP/S[//VP[//NN][//PP]/NP]//VBN \\
\hline TreeBank & TQ13 & ///S//VP//PP[//NP//VBN]//IN \\
\hline TreeBank & TQ14 & ///S//VP//PP[//NN]///NP[//CD]//VBN]//IN \\
\hline TreeBank & TQ15 & [//S[//VP//PP//NP][//S[//PP//JJ]//VBN]//PP//NP//_NONE_ \\
\hline TreeBank & TQ16 & {$[/ / \mathrm{S} / \mathrm{VP} / \mathrm{NP}[/ / \mathrm{VP} / \mathrm{NP} / / \mathrm{ADJ}][/ / \mathrm{NP}[/ / \mathrm{PP} / / \mathrm{VBN}] / / \mathrm{DT}] / / \mathrm{NN}$} \\
\hline TreeBank & TQ17 & //S[/VP/NP[//ADV]//VBN]//VP \\
\hline TreeBank & TQ18 & $/ / \mathrm{S}[/ / \mathrm{VP} / / \mathrm{NP} / / \mathrm{JJ}] / / \mathrm{NP} / \mathrm{PP} / / \mathrm{ADJ}$ \\
\hline TreeBank & TQ19 & //S[/VP[//NP//JJ]/PP//NN]//V \\
\hline TreeBank & TQ20 & ]//S[/VP[//NN][//PP]//A $] / / A D J$ \\
\hline 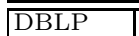 & DQ1 & T//dblp/inproceedings $[/$ title $] /$ author \\
\hline DBLP & DQ2 & //dblp/article[/author][//title]//year \\
\hline DBLP & DQ3 & ///dblp//inproceedings[//cite][//title]//author \\
\hline$\overline{D B L P}$ & DQ4 & //dblp//article[//author][//title][//url][//ee]//year \\
\hline DBLP & DQ5 & //article[//volume][//cite]//journal \\
\hline XMark & $\mathrm{XQ1}$ & ///item[/location]/description//keyword \\
\hline XMark & $\mathrm{XQ2}$ & //people//person[//address/zipcode]/profile/education \\
\hline XMark & $\mathrm{XQ3}$ & ///item[/location][///mailbox/mail//emph]//description//keyword \\
\hline XMark & $\mathrm{XQ} 4$ & //people//person[//address//zipcode][//id]//profile[//age]//education \\
\hline XMark & XQ5 & //open_auction[//annotation//parlist]//bidder//increase \\
\hline
\end{tabular}

ent characteristics over the TreeBank dataset. For the queries TQ1-TQ5, there is at least one non-leaf node with low frequency in each query. On the contrary, the nodes with low frequencies appear on leaf nodes in the queries TQ6-TQ10. For the queries TQ11-TQ15, all the nodes have high frequencies. We compare TwigFast* with TwigFast on these three types of queries. The results are shown in Fig. 2 , As shown in this figure, TwigFast* achieves better performance than TwigFast on all these three types of queries, and is more than $30 \%$ faster than TwigFast on most queries. The better performance of TwigFast* on the queries TQ1-TQ10 suggests that the forward to end approach can avoid the redundant computation brought by late end. On the other hand, TwigFast* achieves better performance than TwigFast on the queries TQ11-TQ15 mainly because re-test checking approach avoids a large amount of unnecessary calls of get Next.

Performance of answering the queries over different datasets. We first compare TwigFast* with TwigFast over the datasets TreeBank, DBLP and XMark. The queries TQ16-TQ20 over TreeBank dataset mix different characteristics we mentioned above. The results are shown in Fig. 3. As shown in this figure, TwigFast* has better efficiency than TwigFast on all of the queries over the three datasets. Then we compare TwigStack* with TwigStack and TJEssential over the datasets TreeBank, DBLP and XMark. The results are shown in Fig. 4. 


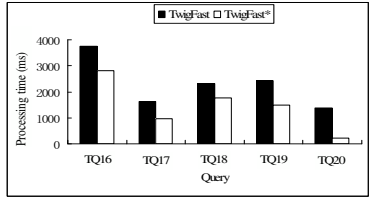

(a) TreeBank

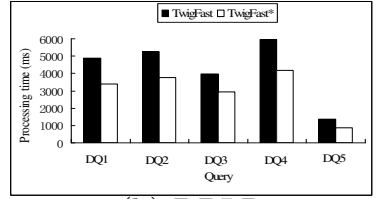

(b) DBLP

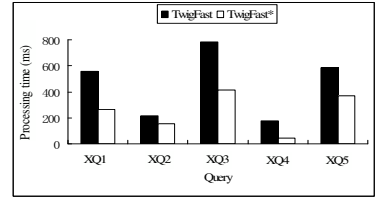

(c) XMark

Fig. 3. TwigFast vs TwigFast*

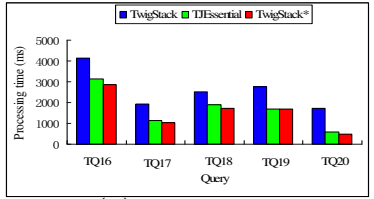

(a) TreeBank

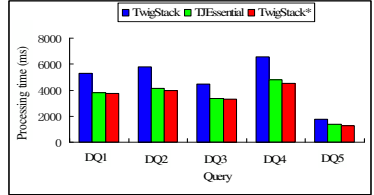

(b) DBLP

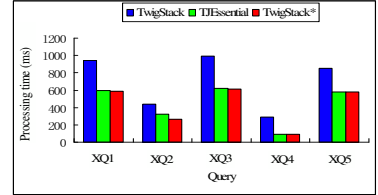

(c) XMark

Fig. 4. TwigStack, TJEssential vs TwigStack*

From the results, we can see that both TJEssential and TwigStack* achieves better performance than TwigStack by resolving the redundant computation even though they use different approaches. Additionally, TwigStack* is a littler faster than TJEssential because TwigStack* can avoid some redundant computation that TJEssential can not avoid and TwigStack* imposes less overheads.

\section{Conclusion}

We presented the approaches re-test checking and forward-to-end, which can be easily applied to both holistic one-phase and two-phase twig pattern matching algorithms that are based on TwigStack, to resolve the redundant computation in getNext. Two algorithms TwigFast* and TwigStack* were presented. The better performance of our algorithms has been verified in our experiments.

\section{References}

1. http://www.cs.washington.edu/research/xmldatasets/

2. Bruno, N., Koudas, N., Srivastava, D.: Holistic twig joins: optimal XML pattern matching. In: SIGMOD Conference, pp. 310-321 (2002)

3. Jiang, Z., Luo, C., Hou, W.-C., Zhu, Q., Che, D.: Efficient processing of XML twig pattern: A novel one-phase holistic solution. In: Wagner, R., Revell, N., Pernul, G. (eds.) DEXA 2007. LNCS, vol. 4653, pp. 87-97. Springer, Heidelberg (2007)

4. Li, G., Feng, J., Zhang, Y., Zhou, L.: Efficient holistic twig joins in leaf-to-root combining with root-to-leaf way. In: Kotagiri, R., Radha Krishna, P., Mohania, M., Nantajeewarawat, E. (eds.) DASFAA 2007. LNCS, vol. 4443, pp. 834-849. Springer, Heidelberg (2007)

5. Li, J., Wang, J.: Fast matching of twig patterns. In: Bhowmick, S.S., Küng, J., Wagner, R. (eds.) DEXA 2008. LNCS, vol. 5181, pp. 523-536. Springer, Heidelberg (2008)

6. Schmidt, A., Waas, F., Kersten, M., Florescu, D., Manolescu, I., Carey, M., Busse, R.: The XML benchmark project. Technical Report INS-R0103, CWI (April 2001) 